\title{
(17) निया \\ Efficacy of intra-articular hyaluronic acid injections in the rehabilitation programme of Knee Osteoarthritis
}

\author{
ONU Ilie ${ }^{1,2^{*}}$, MATEI Daniela ${ }^{1}$, GALACTION Anca ${ }^{1,2}$
}

Corresponding author: Ilie Onu, E-mail: ilie.onu@tuiasi.ro

1. Department of Biomedical Sciences, Faculty of Medical Bioengineering,

University of Medicine and Pharmacy "Grigore T. Popa" Iasi, Romania

2. "Cristofor Simionescu" Faculty of Chemical Engineering and Environmental Protection, from "Gheorghe Asachi” Technical University of Iasi, Romania

\begin{abstract}
Knee osteoarthritis (KOA) is a disease of wear and tear, leading to disability and ultimate surgical treatment. KOA has multiple etiologies and is considered a disorder of physiological processes that is manifested by progressive lesions of articular cartilage caused by chondrolysis, synovial membrane inflammation and subchondral osteophytes production. Modalities that limit the progression of the KOA and rehabilitation are a constant interest for clinicians and medical researchers. Various physical therapies and pharmacological strategies are currently used for pain management, with no clear benefit in terms of inhibiting disease progression. In this context, the use of exogenous hyaluronic acid (HA) (that is a vital component of the synovial fluid and involved in lubricating all synovial joints) has been explored as viable alternative to alleviate knee pain and temporarily restore knee function. In the following, we will review the use of HA injections associated with the physiotherapeutic recovery plan in the treatment of KOA. The therapeutic effects of the HA is still a matter of debate in the field, with no consensus being reached so far and thus, difficult to evaluate. However, much research has to be done to understand the therapeutic efficacy of HA alone or in combination with physical therapies and the role that other factors may play in unraveling its beneficial effects on the KOA.
\end{abstract}

Key words: knee, osteoarthritis, hyaluronic acid, physiotherapy, rehabilitation,

\section{Introduction}

Osteoarthritis (OA) is a chronic condition of the locomotor system that affects the joints and spine in the middle-aged population, leading to disability. Symptomatic knee injury affects 250 million people worldwide, both men and women (1). Progression of the disease is associated with age and ultimately leads to total loss of articular cartilage. Unfortunately, non-surgical and physical therapies have been shown to have limited long-term efficiency and high toxicity generated by extended use of medication.

\section{Osteoarthritis clinical and paraclinical evaluation}

The main manifestations of the knee OA (KOA) are defined by the slow but progressive destruction of articular cartilage, along with changes in the subchondral bone surface and synovia. KOA is a disease with multiple etiologies in which articular "wear and tear" is not the main cause as thought, but rather a disorder of physiological processes that is manifested by progressive lesions of articular cartilage and other structures (2). In terms of KOA classification, it may be primitive where trigger factors are not fully known; and secondary KOA in which local and / or systemic trigger factors are known. The basis of KOA processes is the aging of biomechanical systems, degradation being caused by chondrolysis, inflammation of the synovial membrane, and subchondral osteophytes production. Early physiopathological KOA is characterized by increased hydration of the cartilage matrix, leading to loosening the collagen network and altering the distribution of proteoglycans. The cartilage becomes irregular and the first cracks and tears appear. Progression of the disease is correlated with increased stress in chondrocytes, loss of elasticity and exposure to degrading enzymes (2). Later changes of KOA are characterized by osteophytes formation, subchondral bone cysts, and cartilage ulcerations from focal to diffuse areas. Late changes also have other features such as: soft tissue damage around the joints, proximal muscle weakness, abnormal ligament laxity and inflammatory infiltrations in the synovium. The inflammatory and destructive factors in KOA are: the presence of adipokines (3), IL-1 and TNF- $\alpha$ cytokines, which regulate matrix metalloproteinase (MMP) gene 
expression, which participates in cartilage destruction and inhibits repair processes; proinflammatory cytokines IL-6, IL-8, IL-11, IL-17 and IL-4, IL-10, IL-13 anti-inflammatory cytokines and the presence of MMP degradation enzymes (2, 3). The diagnosis of KOA is classified according to the Kellgren and Lawrence (KL) evaluation system, which is a widespread radiological diagnosis tool (4). Antero-posterior radiographic images are performed to realize the stage of KOA on a zero to four scale; zero for the healthy knee and four for maximum narrowing of the joint space. Clinical diagnosis of KOA contains three important symptoms: short term knee pain, morning stiffness and functional limitation. Clinical signs are: crepitation, limited range of motion and bone deformation (5). Functional examination is largely based on measuring the Range Of Motion (ROM) for the flexion and extension of the knee, which can be performed both, actively and passively (6). The muscular force of the extensor of the knee is another key element in KOA evaluation. The phenomenon is known as "arthrogenic inhibition" of quadriceps muscle, inhibition resulting in increased muscle weakness being closely related to disease progression (7). Chronic pain in KOA is a complex that includes important nociceptive components, and neuropathic elements (8). Another consideration is functional gait evaluation of KOA by observing abnormal movements, distorted or compensated indirectly, that may indicate instability ligament and muscle failure.

\section{Osteoarthritis treatment and recovery}

KOA treatment and rehabilitation is still a subject of debate discussed by multiple disciplines, each developing treatment guidelines specific to the methods used. Unfortunately, no cure for KOA has yet been found, however many ways of preventing and limiting the progression of disease have been explored. Primary or secondary KOA has the same pharmacological, non-pharmacological, physical therapy, rehabilitation, and surgical treatment. In 2014 Osteoarthritis Research Society International has published a guide to KOA non-surgical treatment based on the literature and scientific evidence (9). The general guidelines of the study are directed to non-surgical, non-pharmacological and physical therapy, treatment and rehabilitation, focusing on patient education and self-care.
Adequate treatment modalities suitable for all patients with KOA was considered topical and oral NSAIDs (COX-2), physical therapy exercise and Duloxetine was recommended to patients with multiple arthrosis without other complications. Treatments with moderate efficacy were also considered: chondroitin, glucosamine, intra-articular infiltration with Hyaluronic Acid (HA) and TENS electroanalgesia (10). KOA treatment recommended by American College of Rheumatology was divided into two branches: non-pharmacological and pharmacological approaches. Non-pharmacological treatment includes weight loss, physical therapy exercises, thermotherapy, TENS electroanalgesia, rehabilitation, patellar Kinesio Taping ${ }^{\circledR}$, orthopedic insoles and psychosocial programs. If no results are obtained with pharmacological treatment with: NSAIDs (COX-1 and COX-2), Acetaminophen, corticosteroids injections, HA injections, Tramadol, Duloxetine, the next step are opioids in the desire to delay surgical interventions (11). American Academy of Orthopedic Surgeons recommends, as a pharmacological treatment for KOA, administration of oral NSAIDs, local NSAIDs and Tramadol. Moreover, in 2013 they did not recommend treatments with HA intra-articular injectionos (12). European League Against Rheumatism published a clinical guide using available scientific evidence and an interdisciplinary consensus for several treatment modalities (13). The strong recommendation was: weight loss, NSAIDs, analgesics, food supplements, educating the patients using rehabilitation physical therapy exercise, intra-articular injection of steroids, topical / periarticular NSAIDs application, and opioids, intra-articular HA injection, orthopedic insoles, articular lavage and patellar Kinesio Taping $^{\circledR}$ (14).

\section{Hyaluronic acid in knee osteoarthritis}

$\mathrm{HA}\left(\mathrm{C}_{33} \mathrm{H}_{54} \mathrm{~N}_{2} \mathrm{O}_{23}\right)$ is a natural carbohydrate found in the human body, with a molecular weight $846,786 \mathrm{~g}$ / mol. HA is an amorphous, glassy substance and is part of the class of glycosaminoglycans or acid mucopolysaccharide compounds, with the effect of filling the extracellular spaces between the collagen fibers. HA behavior in biological structures is to attract water, lubricate intracellular structures and give "volume", forming a gelatinous matrix with which the elastin and collagen fibers are coagulated and aligned together (15). 
HA is present in most connective tissues and especially in synovial fluid (16). HA degradation is a step-by-step process that can occur through enzymatic or non-enzymatic reactions. A reduction in molecular HA weight by degradation or slowing of synthesis affects physical and chemical properties such as tissue volume, viscosity and elasticity. HA is a molecule found in abundance in the knee joint and especially in synovial fluid, but also in articular cartilage. The knee joint is a harsh bio-mechanical environment because it is avascular, aneural and alimphatic, where the synovial fluid serves as a lubricant with special rheological properties. Synovial fluid also has the property of clearing free radicals and regulating intracellular activity and proteins binding. Progression of KOA is closely related to the loss of synovial fluid lubrication function. This process is a consequence of depolimerization of endogenous HA with high molecular weight (6500-10900 kDa) and his transformation in a low molecular weight (2700$4500 \mathrm{kDa}$ ). HA with low molecular weight leads to a synovial fluid with much reduced mechanical and visco-elastic properties. Injection of a high molecular weight exogenous HA may be used in the KOA to limit the loss of properties of synovial fluid due to endogenous depolimerisation of natural HA. Exogenous HA does not replace or restore endogenous HA but its presence in the joint induces an improvement in the symptoms of KOA over a period of several months (17). This is possible due to the synthesis of glycosaminoglycan and/ or synthesis of proteoglycan, thus synovial fluid maintaining its visco-elastic properties. Exogenous HA has also an important antiinflammatory effect which, through secondary mechanisms, will reduce pain. FDA-approved (Food and Drug Administration) HA injected products have various physico-chemical characteristics that make a product more efficient/competitive than the other. The molecular weight is the main element targeted by clinicians, and this can range from 500 up to 6000 $\mathrm{kDa}(17)$. As a general rule, the higher the molecular weight, the longer the therapeutic efficacy. The source of animal and non-animal HA origin is another criteria for the selection, along with the molecular structure (linear, crosslinked or in combination), degree and crosslinking method, the concentration that can range between $0.8-30 \mathrm{mg} /$ $\mathrm{ml}$, and dose of injected volume $(0.5-0.6 \mathrm{ml})$. For pain evaluation of KOA, the most commonly used scales are VAS (visual analogue scale) and WOMAC (Western Ontario and McMaster Universities Osteoarthritis Index) in which pain severity is correlated with intra-articular changes. The KL radiological score is associated with the presence of osteophytes, cartilage lesions, which is the independent predictor for the VAS scale (18).

The efficacy and safety of HA-based products has been constantly discussed and analyzed over the past two decades. Although studies have shown medium and long-term improvements in the use of injected HA in the knee, the disease progression has not been stopped, ending in total knee arthroplasty. The efficacy of Durolane ${ }^{\circledR}$, that has very high molecular mass $(100,000 \mathrm{kDa})$, was questioned by Altman (19) and colleagues from the WOMAC score in a 2004 study. Hylagan ${ }^{\circledR}(6000 \mathrm{kDa})$ is disputed by Lundsgaard et al. (20) showing that there are no notable benefits in relation to physiological saline injection. In another study from 2005, Karatosun (21) shows that there are no differences in high and low molecule HA injections in advanced KOA, and both groups benefiting from the same improvements at the last assessment. Neustadt et al. (22) demonstrated that Orthovisc $\AA$ provides a symptomatic improvement in the advanced KOA stages, and favorable clinical results were obtained at an early and moderate stage according to the studies of Day et al. (23) and Brandt et al. (24). Berenbaum et al. (25) compared the results obtained by injecting GO-ON (800-1500 kDa) and Hyalgan ${ }^{\circledR}$ and with GO-ON, they obtained statistically significant improvements in KOA symptoms over a period of 6 months. Day et al. have demonstrated that HA injection is effective, safe and well tolerated by patients, and have achieved positive results both in function and in pain decreasing in low - moderate $\mathrm{KOA}$, that is confirmed by Brandt et al. (24), Neustadt et al. (20) evaluated and demonstrated the effectiveness of Orthovisc ${ }^{\circledR}$ in the treatment of KOA on the final radiological stage (17). Two other studies of the efficacy of Hyalgan ${ }^{\circledR}$ have shown that the effects of intra-articular injection are similar to Naproxen treatment, taken continuously over a 26week period of time (17). According to the Journal of the American Academy of Orthopaedic Surgeons, Naproxen is the most effective NSAID used in the treatment of KOA with a powerful analgesic effect, but its long-term use develops characteristic 
NSAID's side effects (26). KOA is a surgical endwear disease. Intra-articular HA injection in the knee opens new horizons and new possibilities to limit the progression of the disease, thus treating the causes of joint damage. It is known that HA only lasts 2-3 days in the joint, however its chondroprotective effects are seen over the several weeks post-injection (27). HA provides the synthesis of extracellular matrix proteins such as collagen type II and increased proteoglycan and glycosaminoglycan synthesis $(28,29,30)$. HA reduces proteoglycan loss in cartilage and apoptosis of chondrocytes (28). Intra-articular HA stimulates the endogenous synthesis of HA ensuring joint lubrication (31). Also, by decreasing local production of cytokines, HA reduces the degradation of joint and decreases the generation of pain mediators (28). By binding to CD44 nociceptors, HA reduces prostaglandin and cyclooxygenase (COX-2) production, and by activating opioid receptors, alleviates pain through modification of pain pathways (31). Therefore, HA has shown efficacy in the treatment of KOA by its anti-inflammatory, chondroprotective and analgesic effects. A conducted study made in 2015 by the European Society of Sports Traumatology, Knee Surgery, Arthroscopy was conducted to assess the effectiveness of recovery through therapeutic exercise in combination with injected HA in moderate KOA. The study was prospective, randomized trial controlled, blinded, with three patient groups over a 6-month period. Group 1 only received HA injected two weeks, Group 2 only performed individualized exercise up to 20 sessions in one month. Group 3 simultaneously received both types of treatment in combination. The evaluation was done using the WOMAC scale and active motion domain (AROM), patients passing through successive series of assessments at 1,3 and 6 months after the treatment session. All patient groups experienced improvements from one month after treatment, and group 3 experienced a significant improvement over the first group. In the second group from the second to the sixth month of treatment, WOLMAC score was modified, showing the symptoms worsening (stiffened joint, pain and the gradual loss of mechanical function). From the AROM evaluation perspective, there were no differences between groups during the 6 months of monitoring. The authors conclude that HA injected therapy combined with adapted medical exercise has greatly improved pain one month after treatment. Improving knee function and limiting KOA progression with injected $\mathrm{HA}$ is due to the counterbalance of the negative effect of inflammatory mediators that are involved in chronic synovitis, cartilage destruction in bone remodeling and soft tissues (32). A multicenter study was conducted in the United States, to determine the effectiveness of physical therapy associated with the injection of HA to treat and recovery KOA, from the perspective of clinical utility and costs. The combined therapy program for recovery KOA, was composed by HA injectable and therapeutic physical exercises that included: muscle toning, flexibility exercises and proprioception. The design of the study whas to track 553 patients with symptomatic KOA for 8 weeks in 27 USA specialized centers, patients who had undergone previous but not effective pharmacological treatments. Physical therapy was performed under the guidance of physiotherapists throughout the 2-3 sessions in an week. The result was quantificated by reducing the knee pain by $59 \%$ in all evaluated patients. The WOLMAC score was improved with values ranging from $44 \%$ to $51 \%$, with patients experiencing a significant reduction in specific symptomatology, even in KL3-KL4 stages. The combined therapy program is said to be cost-effective ratio over 2 years of monitoring, clearly delaying the surgical treatment of the knee. The authors believe that rehabilitation through physical therapy programs and HA injections acts through a synergistic handler using different therapeutic mechanisms that can limit the progression of KOA in the medium term and with a good cost-effectiveness ratio (33).

\section{For the future}

Clinical studies and meta-analyses have sometimes reported contradictory results on the safety and effectiveness of HA injections for KOA. Due to these confounding results, KOA treatment and rehabilitation guidelines differ from one author to another and from one society to another, sometimes uncertain recommendations are made and often objectionable. From the perspective of side effects, when compared to corticosteroids and saline solution, HA does not appear to have higher side effects. Controlling the symptoms on medium and long term in mild and moderate KOA, HA provides better and more stable outcomes than corticosteroids 
and rehabilitation physical therapy improve the function of knee. The clinical response differs from one solution to another, rheological parameters also can differ, and the assessment of the product classes has to be done individually, because the universal evaluation may not be adequate. Physical therapy rehabilitation programs optimized on patient are much more effective than general - homemade treatment programs and in combination with TENS electroanalgesia, VAS and WOLMAC score are improved.However, additional research is needed on the HA's chondroprotective mechanisms, in larger group samples and longer periods of observation, in order to clarify the therapeutic effects and the safety of HA for widespread use. It would also be interesting to study various ways of controlling the evolution of the disease and its side effects. Exploiting the potential of HA's treatments with different molecular compositions in combination with physical therapy, clinicians could improve the KOA's overall treatment guidelines. More in-depth studies would be required based on a clear and homogeneous methodology, taking into account rheological behavior, molecular weight, injected amount, HA origin, disease status, comorbidities and combinations with other drugs. Nevertheless, the quality of these studies could be influenced by data processing method and that should be homogeneous and the collection, processing, analysis, data interpretation and generation of conclusions should be in consensus. More comprehensive studies are needed, in which HA injected therapy in particular could be studied alongside, rehabilitation physical therapy, TENS, LED, LASER, cold-warm therapy, ultrasound, TECAR, etc.

\section{References}

1. O'Neill TW, McCabe PS, McBeth J. Update on the epidemiology, risk factors and disease outcomes of osteoarthritis. Best Pract Res Clin Rheumatol. 2018 Apr; 32(2):312-326.

2. Ali A, Rosenberger L, Weiss RT, Milak C, Perlman A. Massage Therapy and Quality of Life in Osteoarthritis of the Knee: A Qualitative Study. Pain Med. 2017 Jun; 18(6): 1168-1175.

3. Wang T, He C. Pro-inflammatory cytokines: The link between obesity and osteoarthritis, Cytokine Growth Factor Rev. 2018; 44:38-50.

4. Kohn MD, Sassoon AA, and Fernando ND. Classifications in Brief: Kellgren-Lawrence Classification of Osteoarthritis, Clin Orthop Relat Res. 2016; 474(8): 1886-1893.
5. Zhang W, Doherty M, Peat G, Bierma-Zeinstra MA, Arden NK, Bresnihan B, Herrero-Beaumont G, Kirschner S, Leeb BF, Lohmander LS, Mazières B, Pavelka K, Punzi L, So AK, Tuncer T, Watt I, Bijlsma JW, EULAR evidence-based recommendations for the diagnosis of knee osteoarthritis. Ann Rheum Dis. 2010; 69:483-489.

6. Hsu H, Siwiec RM, Knee Osteoarthritis, Treasure Island (FL): StatPearls Publishing. 2018.

7. Rafsanjani $\mathrm{H}$, Khademi-Kalantari K, Rezasoltani A, Naimi SS, Ghasemi M, Jaberzadeh S, Immediate effect of common peroneal nerve electrical stimulation on quadriceps muscle arthrogenic inhibition in patients with knee osteoarthritis. J Bodyw Mov Ther. 2017 ;21(4):879-883.

8. Filatova E, Turovskaya E, Erdes S, et al. AB0314 Neurogenic Mechanisms of Chronic Joint Pain in Patients with Rheumatoid Arthritis and Knee Osteoarthritis. Annals of the Rheumatic Diseases 2014;73:908.

9. Guidelines | Osteoarthritis Research Society International (OARSI) 2019 http://www.oarsi.org/education/oarsi-guidelines

10. McAlindon TE, Bannuru RR, Sullivan MC, Arden NK, Berenbaum F, Bierma-Zeinstra SM, Hawker GA, Henrotin Y, Hunter DJ, Kawaguchi H., Kwoh K, Lohmander S, Rannou F, Roos EM, Underwood $\mathrm{M}$, OARSI guidelines for the non-surgical management of knee osteoarthritis. Osteoarthritis Cartilage. 2014 ;22(3):363-88.

11. Hochberg MC, Altman RD, April KT, Benkhalti M, Guyatt G, McGowan J, Towheed T, Welch V, Wells G, Tugwell P. American College of Rheumatology, American College of Rheumatology 2012 Recommendations for the Use of Nonpharmacologic and Pharmacologic Therapies in Osteoarthritis of the Hand, Hip, and Knee. Arthritis Care Res (Hoboken). $2012 ; 64(4): 465-74$.

12. Lozada CJ, Diamond FS, Osteoarthritis Guideline 2018

https://emedicine.medscape.com/article/330487guidelines\#g3.

13. Pendleton A, Arden N, Dougados M, Doherty M, Bannwarth B, Bijlsma JW, Cluzeau F, Cooper C, Dieppe PA, Günther KP, Hauselmann HJ, HerreroBeaumont G, Kaklamanis PM, Leeb B, Lequesne M, Lohmander S, Mazieres B, Mola EM, Pavelka K, Serni U, Swoboda B, Verbruggen AA, Weseloh G, Zimmermann-Gorska I. EULAR recommendations for the management of knee osteoarthritis: report of a task force of the Standing Committee for International Clinical Studies Including Therapeutic Trials (ESCISIT). Ann Rheum Dis. 2000 ;59(12):936-44. 
14. Fernandes L, Hagen KB, Bijlsma JW, Andreassen $\mathrm{O}$, Christensen P, Conaghan PG, Doherty M, Geenen R, Hammond A, Kjeken I, Lohmander LS, Lund H, Mallen CD, Nava T, Oliver S, Pavelka K, Pitsillidou I, da Silva JA, de la Torre J, Zanoli G, Vliet Vlieland TP; European League Against Rheumatism (EULAR). EULAR recommendations for the non-pharmacological core management of hip and knee osteoarthritis. Ann Rheum Dis. 2013 ;72(7):1125-35.

15. Matarasso SL, Understanding and Using Hyaluronic Acid Aesthetic Surgery Journal, Volume 24, Issue 4, 1 July 2004, Pages 361-364.

16. PubChem is an open chemistry database at the National Institutes of Health (NIH) 2019 https://pubchem.ncbi.nlm.nih.gov/compound/24847 767

17. Bowman S, Awad ME, Hamrick MW, Hunter M, Fulzele $\mathrm{S}$, Recent advances in hyaluronic acid based therapy for osteoarthritis. Clin Transl Med. 2018 16;7(1):6.

18. Serban O, Porojan M, Deac M, Cozma F, Solomon C, Lenghel M, Micu M, Fodor D. Pain in bilateral knee osteoarthritis - correlations between clinical examination, radiological, and ultrasonographical findings. Med Ultrason. 2016;18(3):318-25.

19. Altman RD, et al. Efficacy and safety of a single intra-articular injection of non-animal stabilized hyaluronic acid (NASHA) in patients with osteoarthritis of the knee. Osteoarthr Cartil. 2004;12(8):642-649.

20. Lundsgaard $C$, et al. Intra-articular sodium hyaluronate $2 \mathrm{~mL}$ versus physiological saline $20 \mathrm{~mL}$ versus physiological saline $2 \mathrm{~mL}$ for painful knee osteoarthritis: a randomized clinical trial. Scand J Rheumatol. 2008;37(2):142-150.

21. Karatosun $\mathrm{V}$, et al. Intra-articular hyaluronic acid compared to exercise therapy in osteoarthritis of the ankle. A prospective randomized trial with longterm follow-up. Clin Exp Rheumatol. 2008;26(2):288-294.

22. Neustadt D, et al. Clinical effects of intraarticular injection of high molecular weight hyaluronan (Orthovisc) in osteoarthritis of the knee: a randomized, controlled, multicenter trial. J Rheumatol. 2005;32(10):1928-1936.

23. Day $\mathrm{R}$, et al. A double blind, randomized, multicenter, parallel group study of the effectiveness and tolerance of intraarticular hyaluronan in osteoarthritis of the knee. J Rheumatol. 2004;31(4):775-782.
24. Brandt KD, et al. Efficacy and safety of intraarticular sodium hyaluronate in knee osteoarthritis. ORTHOVISC Study Group. Clin Orthop Relat Res. 2001;385:130-143.

25. Berenbaum F, Osteoarthritis as an inflammatory disease (osteoarthritis is not osteoarthrosis!) Osteoarthr Cartil. 2013;21(1):16-21.

26. Jevsevar DS, Shores PB, Mullen K, Schulte DM, Brown GA, Cummins DS. Mixed Treatment Comparisons for Nonsurgical Treatment of Knee Osteoarthritis: A Network Meta-analysis. J Am Acad Orthop Surg. 2018 ;26(9):325-336.

27. Fakhari A, Berkland C. Applications and emerging trends of hyaluronic acid in

1. tissue engineering, as a dermal filler and in osteoarthritis treatment. Acta Biomater 2013;9:7081-92

28. Altman RD, Manjoo A, Fierlinger A, Niazi F, Nicholls M, The mechanism of action for hyaluronic acid treatment in the osteoarthritic knee: a systematic review. BMC Musculoskelet Disord 2015;16:321

29. Altman, RD, Dasa V, and Takeuchi J, Review of the Mechanism of Action for Supartz FX in Knee Osteoarthritis. Cartilage. 2018; 9: 11-20.

30. Chen, L, Xue J, Zheng Z, et al. Hyaluronic Acid, an Efficient Biomacromolecule for Treatment of Inflammatory Skin and Joint Diseases: A Review of Recent Developments and Critical Appraisal of Preclinical and Clinical Investigations. International Journal of Biological Macromolecules. 2018; 116: 572-584.

31. Nicholls, M, Fierlinger A, Zaizi F, et al, The Disease Modifying Effects of Hyaluronan in the Osteoarthritic Disease State. Clinical Medicine Insights: Arthritis and Musculoskeletal Disorders. 2017; 10: 1-10.

32. Saccomanno M, Donati F, Careri S, Bartoli M, Severini G, Milano G, Efficacy of intra-articular hyaluronic acid injections and exercise based rehabilitation programme, administered as isolated or integrated therapeutic regimens for the treatment of knee osteoarthritis. Knee Surg Sports Traumatol Arthrosc. 2016 May;24(5):1686-94.

33. Miller L, Block J. An 8-Week Knee Osteoarthritis Treatment Program of Hyaluronic Acid Injection, Deliberate Physical Rehabilitation, and Patient Education is Cost Effective at 2 Years Follow-up: The OsteoArthritis Centers of America SM Experience. Clin Med Insights Arthritis Musculoskelet Disord. 2014;7: 49-55. 